TecnoHumanismo. Revista Científica

Setiembre - Noviembre 2021

Volumen 1 / No.3

ISSN: $2710-2394$

pp. $276-293$

https://doi.org/10.53673/th.v2i1.98

https://tecnohumanismo.online

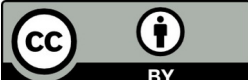

\title{
Proceso logístico efectivo para la generación de valor en las empresas del estado.
}

Effective logistics process for the generation of value in state companies.

Processo logístico eficaz para geração de valor em empresas estatais.

\section{ARTÍCULO GENERAL}
Domingo Hernández Celis
dr.domingohernandezcelis@,gmail.com
https://orcid.org/0000-0002-9759-4436
Universidad Nacional Federico Villareal
Lima - Perú

\author{
Hortencia Valderrama Torre \\ hortenciavalderrama@gmail.com \\ https://orcid.org/0000-0003-0770-5698 \\ Universidad Nacional Federico Villareal \\ Lima - Perú
}

Recibido 07 de Abril 2021 | Arbitrado y aceptado 19 de Junio 2021 | Publicado en 03 Setiembre 2021

\begin{abstract}
RESUMEN
La investigación denominada: El proceso logístico efectivo y la generación de valor en las empresas del Estado; es un aporte académico y profesional, cuyo problema se ha identificado en la deficiente generación de valor en las entidades mencionadas. Esta problemática se concreta en la pregunta general siguiente: ¿Cómo influye el proceso logístico efectivo en la generación de valor de las empresas del estado? Sobre dicha problemática se tiene la alternativa de solución correspondiente a través de la hipótesis general: El proceso logístico efectivo influye en grado significativo en la generación de valor de las empresas del estado. En este contexto, en lo referido al propósito del trabajo se tiene el objetivo general siguiente: Determinar la influencia del proceso logístico efectivo en la generación de valor de las empresas del estado. Se concluye que en promedio el $94 \%$ de los encuestados acepta que el proceso logístico efectivo influye en grado significativo en la generación de valor de las empresas del estado, mediante la utilización de un conjunto variado de herramientas.
\end{abstract}

Palabras clave: Proceso logístico efectivo; generación de valor y empresas del Estado.

\section{ABSTRACT}

The research called: The effective logistics process and the generation of value in state companies; it is an academic and professional contribution, whose problem has been identified in the deficient generation of value in the aforementioned entities. This problem is specified in the following general question: How does the effective logistics process influence the generation of value of state companies? On this problem, there is the corresponding solution alternative through the general hypothesis: The effective logistics process influences to a significant degree the value generation of state companies. In this context, regarding the purpose of the work, the following general objective is had: Determine the influence of the effective logistics process in the generation of value of the state companies. It is concluded that on average $94 \%$ of the respondents accept that the effective logistics process has a significant influence on the generation of value of state companies, through the use of a varied set of tools.

Keywords: Effective logistics process; generation of value and state companies.

\section{RESUMO}

A pesquisa intitulou: $O$ processo logístico eficaz e a geração de valor nas empresas estatais; trata-se de uma contribuição acadêmica e profissional, cujo problema foi identificado na deficiente geração de valor nas referidas entidades. Esse problema é especificado na seguinte questão geral: Como o processo logístico eficaz influencia na geração de valor das empresas estatais? Sobre este problema, existe a correspondente alternativa de solução através da hipótese geral: $\mathrm{O}$ processo logístico eficaz influencia de forma significativa a geração de valor das empresas estatais. Nesse contexto, quanto ao objetivo do trabalho, tem-se o seguinte objetivo geral: Determinar a influência do processo logístico efetivo na geração de valor das empresas estatais. Conclui-se que em média $94 \%$ dos respondentes aceitam que o processo logístico eficaz tem influência significativa na geração de valor das empresas estatais, por meio da utilização de um variado conjunto de ferramentas.

Palavras-chave: Processo logístico eficaz; geração de valor e empresas estatais. 


\section{Introducción}

La deficiente generación de valor de las empresas no es solo un problema de las empresas del estado peruano, lo es también de las empresas de otros estados como de las empresas privadas internacionales, tal como se detalla a continuación:

Situación internacional: Según Tellez (2015) durante la década de los años ochenta y noventa, las finanzas corporativas han estado evolucionando hacia la conformación de una filosofía en donde su objetivo principal es el de maximizar el valor de los accionistas. El objetivo maximizar el valor de los accionistas es un mandato redactado de manera sencilla y pareciera ser de fácil interpretación, sin embargo, aspectos sensibles como la relación accionista y empresa hacen necesario profundizar en su entendimiento. El tema de creación de valor a través de su maximización para los accionistas tiene diferentes significados. En el mismo contexto en opinión de Uruburu, (2013) la responsabilidad social corporativa (RSC), ha adquirido en la actualidad un protagonismo fundamental dentro de las políticas globales de dirección de las grandes compañías.

Situación nacional: Para Small (2018) analizar la creación o generación de valor significa conocer la riqueza generada durante periodos económicos de una compañía, dado el nivel de riesgo del sector. Es importante identificarla, ya que el seguimiento financiero de una empresa no solo debe estar enfocado en la generación de utilidades, sino en la rentabilidad generada, la cual se obtiene considerando el nivel de inversión realizado para generar dichos resultados.

Situación específica: Según Quijada (2018), se tiene que las entidades se encontrarán en condiciones de advertir las situaciones en las cuales se produce una vulneración a la normativa de contratación pública.

Problema general; ¿Cómo influye el proceso logístico efectivo en la generación de valor de las empresas del estado?

Problemas específicos: a) ¿Cómo inciden los sistemas modernos de gestión logística como la planificación de los requerimientos de materiales y la planificación de recursos de manufactura o servicios en la generación de valor de las empresas del estado?; b) ¿Cómo influyen las nuevas herramientas de gestión logística como el benchmarking y el outsourcing en la generación de valor de las empresas del estado?; c) ¿Cómo 
inciden los flujos productivos de la cadena logística como el método: justo a tiempo y la tecnología de producción optimizada en la generación de valor de las empresas del estado?; d) ¿Cómo influye la respuesta eficiente al consumidor, la respuesta rápida y la planeación de los recursos de distribución del proceso logístico efectivo en la generación de valor de las empresas del estado?; e) ¿Cómo inciden la distribución física efectiva del proceso logístico efectivo en la generación de valor de las empresas del estado?

\section{Investigaciones nacionales:}

Valderrama \& Salaverry (2014) señalan que el trabajo de investigación tuvo como objetivo determinar el planeamiento estratégico del departamento de logística del Banco de la Nación. En cambio para Corahua y Mendoza (2018) la productividad de la cooperativa está muy por debajo de lo esperado, es decir, existe un problema. En cambio para Huamán y Rodríguez (2018) indica que del estudio se obtuvo una evaluación, conocimiento de la realidad problemática, que nos permite realizar una estimación de las principales necesidades y deficiencias en todo el proceso logístico de los insumos y materiales médicos utilizados para la atención de los usuarios. Para Alcalde, García, Ponce \& Valdivieso (2014) el objetivo principal de la tesis consiste en hacer un análisis y proponer alternativas de solución, para determinar el impacto en la generación de valor de la Empresa Exalmar S.A.A. Todo lo anterior se complementa con Small (2018) quien estable que el aumento del capital invertido año a año refleja que las tres compañías se encuentran en proceso de expansión, realizando inversiones importantes en activos fijos.

Investigaciones internacionales: Alcocer \& Knudsen (2018) establecen que las medidas propuestas para erradicar los problemas detectados constituyen la base para trazar alternativas o estrategias de mejora, en función de lograr un desempeño efectivo de estos procesos logísticos de las líneas de presillado de la Empresa Gráfica de Villa Clara, que contribuya al aumento de sus resultados productivos y económicos. Bohórquez y Puello (2013) establecen que el trabajo permitió poner en práctica todos aquellos conocimientos adquiridos a lo largo de la carrera profesional cursada, incentivando el uso de herramientas para la solución de problemas empresariales. Por su parte Rubio (2018) indica que el sistema de logística inversa tiene un proceso y se realiza en varias funciones con procedimientos específicos que cada día es mejorado en las empresas. De otro lado Marqués (2017) señala que las conclusiones evidencian la confirmación de la hipótesis de trabajo planteada, ya que las grandes empresas españolas sí han avanzado en 
incorporar la sostenibilidad en sus organizaciones. Para Chang, Gamarra \& López (2019) la empresa Backus presenta un crecimiento sostenido de sus ingresos. Backus pertenece al grupo cervecero mundial número 1 en el mundo. Backus presenta un importante nivel de rentabilidad, medido a través del ROE y adecuados márgenes.

\section{Bases teóricas}

Proceso logístico efectivo: Para Parodi (2017) la gestión logística cumple una función gerencial de integración de los procesos de planificación, control y distribución, que involucra además de la distribución física, es decir, del almacenamiento y el transporte, así como el abastecimiento oportuno de los bienes y servicios, requeridos por la organización. En cambio según Silva (2017) el proceso logístico está conformado por todas aquellas actividades que ayudarán a la empresa a administrar de manera eficiente sus materias primas, así como sus productos terminados, la producción y distribución de sus productos mediante una programación y rutas idóneas, en el menor tiempo posible. Mora (2017) establece que el proceso de adquisición es aquel mediante el cual la empresa obtiene los materiales y/o productos necesarios para continuar con la manufactura de sus productos, con la comercialización de sus productos y/o con sus actividades administrativas. Este proceso está generalmente asociado al proceso de compras, ya que a través de éste la empresa obtiene lo necesario para continuar con sus actividades. En opinión de Velásquez (2016) se tiene que el proceso de adquisición con el soporte de información transparente y de fácil acceso, sumado al procedimiento adecuado en función a las características del producto o servicio, permitirá generar valor agregado al mejor precio de mercado, tanto al comprador como al proveedor. Entre tanto para Ramírez (2018) la importancia de la logística se establece en la suficiencia que tenga al resolver de manera eficaz y eficiente las dificultades.

Generación de valor: Según Bonmatí (2018) entendemos por valor de la empresa el valor del conjunto de elementos, materiales, inmateriales y humanos que integran o constituyen la empresa. Por su parte Viscarri, (2019) considera que las empresas se enfrentan a la complicada tarea de generar valor para el cliente en un entorno de continuos cambios en los que el consumidor es cada vez más exigente. Según Álvarez (2018) el actual entorno presenta realidades como la virtual inexistencia de fronteras, la constante innovación tecnológica, la evolución de las comunicaciones, la movilidad de bienes y servicios, la rápida transferencia de capitales y su efecto sobre la volatilidad en las 
economías. En la profundización del tema se tiene a Orna (2015) el mismo que indica que las empresas deben ser flexibles para responder rápidamente a los cambios de la competencia.

Empresas del estado: Melgarejo (2019, junio, 09) considera que las 34 empresas del Estado tenían S/ 618 millones para invertir hasta abril, pero solo han utilizada S/ 347 millones, dejando S/ 271 millones sin ejecutar. Essalud que más ha dejado de invertir, con S/ 92 millones hasta el mes de abril. En complemento Sayago (2019, marzo, 29)indica que las empresas del Estado comprendidas en el ámbito del Fondo Nacional de Financiamiento de la Actividad Empresarial del Estado impulsan iniciativas que generan valor. De otro lado, según el Portal El Peruano (2017, noviembre, 22) uno de los activos del gobierno del presidente Pedro Pablo Kuczynski es su política de modernización y simplificación administrativa del Estado. Por otro lado según el Portal de FONAFE (2019, junio, 28), el modelo peruano aplicado a la gestión de las empresas públicas en el Perú, será replicado en Argentina y Brasil. Cobeñas (2011, junio, 13) considera que tenemos 34 empresas públicas teniendo pendiente la inversión en Educación, Salud, Defensa, Seguridad Ciudadana e inclusión social.

\section{Marco conceptual de la investigación:}

Materials Requirements Planning (MRP-I): Planificación de los requerimientos de materiales: Según Ramírez (2018) señala que el MRP (Material Requirements Planning) significa, en español, Planificación de Requerimientos de Material y se trata de un sistema de planificación utilizado en la gestión de los procesos de fabricación que nos permite asegurar que dispondremos del material necesario para nuestra producción a tiempo.

\section{Resource Requirements Plan of. Manufacturing (MRP II): Planificación de} recursos de manufactura: Para Ramírez (2018) señala que la planificación de recursos de manufactura es una herramienta logística que utiliza datos de una previsión de ventas futuras hasta el tiempo y los recursos necesarios para fabricar un producto.

Just in time (JIT): Método: justo a tiempo: En opinión de Ramírez (2018) señala que el justo a tiempo, es una metodología cuyo objetivo es contar con la cantidad necesaria de producto, en el momento y lugar justo, eliminando cualquier desperdicio o elemento que no aporte al desarrollo empresarial. 
Optimized Production Technology (OPT): Tecnología de producción optimizada: Para Ramírez (2018) señala que la tecnología de producción optimizada es un método de gestión de flujos de producción de una determinada empresa.

Quick response (QR): Respuesta rápida: Para Ramírez (2018) la respuesta rápida en logística es una metodología de optimización.

Efficient consumer response (ECR): Respuesta eficiente al consumidor: Para Ramírez (2018) señala que el efficient consumer response se refiere a las respuestas que debe dar la empresa a sus clientes, consumidores o usuarios en todo lo que pidan en términos de productos, servicios, reclamos, etc.

Distribution Resource Planning (DRP): Planeación de los Recursos de distribución: Según Ramírez (2018) señala que la respuesta eficiente al consumidor es el trabajo colaborativo entre los clientes y los proveedores

Visión empresarial: Según Bonmatí (2018) la visión empresarial es lo que desea ser una organización en el mediano y largo plazo; siempre se enmarca en superar largamente la misión y se apuesta por trascender frente al resto de empresas.

Misión empresarial: Según Bonmatí (2018) la misión empresarial es el quehacer actual de la organización; es decir involucra las actividades, procesos y funciones que realiza. Dicha misión se concreta con el logro de las metas y objetivos empresariales.

Valores empresariales: Según Bonmatí (2018) los valores empresariales son un conjunto de principios en los cuales se basa el accionar de los directivos, funcionarios y trabajadores de la organización. Dichos valores son una suerte de columnas vertebrales de la manifestación del capital humano de la entidad.

Mejora continua empresarial: Según Bonmatí (2018) la mejora continua empresarial se relaciona con varios aspectos de las organizaciones como la creatividad e innovación sobre los recursos, actividades, procesos y procedimientos empresariales.

Competitividad empresarial: Según Bonmatí (2018) la competitividad empresarial es posible por la imposición de una empresa frente a las demás de su sector. 


\section{Justificación}

Este trabajo se justifica por cuanto contribuirá en forma efectiva a la solución del problema de la cadena de valor mediante el proceso logístico efectivo, es decir el proceso logístico alineado a las metas, objetivos, misión y visión de las empresas del estado.

Objetivo general: Determinar la influencia del proceso logístico efectivo en la generación de valor de las empresas del estado.

Objetivos específicos: a) Establecer la incidencia de los sistemas modernos de gestión logística como la planificación de los requerimientos de materiales y la planificación de recursos de manufactura o servicios en la generación de valor de las empresas del estado; b) Determinar la influencia de las nuevas herramientas de gestión logística como el benchmarking y el outsourcing en la generación de valor de las empresas del estado; c) Establecer la incidencia de los flujos productivos de la cadena logística como el método: justo a tiempo y la tecnología de producción optimizada en la generación de valor de las empresas del estado; d) Determinar la influencia de la respuesta eficiente al consumidor, la respuesta rápida y la planeación de los recursos de distribución del proceso logístico efectivo en la generación de valor de las empresas del estado; e) Establecer la incidencia de la distribución física efectiva del proceso logístico efectivo en la generación de valor de las empresas del estado.

Hipótesis general: El proceso logístico efectivo influye en grado significativo en la generación de valor de las empresas del estado.

Hipótesis específicas: a) Los sistemas modernos de gestión logística como la planificación de los requerimientos de materiales y la planificación de recursos de manufactura o servicios inciden en grado significativo en la generación de valor de las empresas del estado; b) Las nuevas herramientas de gestión logística como el benchmarking y el outsourcing influyen en grado significativo en la generación de valor de las empresas del estado; c) Los flujos productivos de la cadena logística como el método: justo a tiempo y la tecnología de producción optimizada inciden en grado significativo en la generación de valor de las empresas del estado; d) La respuesta eficiente al consumidor, la respuesta rápida y la planeación de los recursos de distribución del proceso logístico efectivo influyen en grado significativo en la generación de valor de 
las empresas del estado; e) La distribución física efectiva del proceso logístico efectivo incide en grado significativo en la generación de valor de las empresas del estado.

\section{Materiales y métodos}

Tipo de investigación: Esta investigación es aplicada; por cuanto se propone aplicar el proceso logístico efectivo para solucionar el problema de la generación de valor en las empresas del estado.

Diseño de la investigación: El proceso logístico efectivo y la generación de valor en las empresas del estado; ha tenido en cuenta el diseño no experimental; el mismo que no requiere demostración alguna, pero tampoco es factible de manipulación o control.

Población: El proceso logístico efectivo y la generación de valor en las empresas del estado ha considerado una población estadística de 135 personas.

Muestra: El proceso logístico efectivo y la generación de valor en las empresas del estado ha considerado un muestra de 100 personas.

Área de estudio: El área de estudio ha sido las empresas del estado.

Procedimientos estadísticos: Se han aplicado los siguientes procedimientos estadísticos: a) Correlación de Spearman; y, b) Regresión simple

Aspectos éticos: Para Ojeda, Quintero, y Machado (2017) la ética, como rama de la filosofía, tiene un papel importante en todos los ámbitos, tanto públicos como privados $\mathrm{y}$, especialmente en los relacionados con la investigación científica.

\section{Resultados}

\section{Análisis e interpretación de los resultados de la variable independiente:}

Resultado 1: El 94\% de los encuestados acepta que el proceso logístico efectivo es el conjunto de fases que facilitan las relaciones del movimiento de los productos, tanto externa como internamente, con el objeto de cumplir la misión de las empresas del estado.

Resultado 2: El 94\% de los encuestados acepta que el sistema de planificación de los requerimientos de materiales (MRP-I), procura determinar a priori los materiales que necesitan las empresas del estado para cumplir la misión. 
Resultado 3: El 84\% de los encuestados acepta que la planificación de recursos de manufactura o de suministro (MRP-II), procura generar valor en las empresas del estado.

Resultado 4: El 85\% de los encuestados acepta que el benchmarking es un proceso continuo por el cual se toma como referencia los productos, servicios o procesos de trabajo de las empresas líderes, para compararlos con los de tu propia empresa y posteriormente realizar mejoras e implementarlas.

Resultado 5: E1 94\% de los encuestados acepta que el outsourcing es el proceso en el cual las empresas del estado contratan a otras empresas externas para que se hagan cargo de parte de su actividad o producción y así lograrla misión y generar valor-

Resultado 6: El 96\% de los encuestados acepta que los flujos productivos en la cadena logística serán más adecuados mediante la aplicación de la filosofía del justo a tiempo (JIT), que considera la reducción o eliminación de todo lo que implique desperdicio en las actividades de compras, fabricación, distribución, etc.

Resultado 7: El 78\% de los encuestados acepta que la tecnología de producción optimizada (OPT), es una herramienta para balancear la producción de una empresa mejorando los cuellos de botella y aumentando la capacidad de producción, se basa en la teoría de las restricciones.

Resultado 8: El 94\% de los encuestados acepta que la relación entre la logística de una empresa del estado consumidor o usuario será mejor y ayudará a lograr la misión, si considera la respuesta eficiente al consumidor (ECR).

Resultado 9: El 96\% de los encuestados acepta que en el proceso logístico es imprescindible que el cliente o usuario tenga una respuesta rápida $(\mathrm{QR})$, lo cual ayudará en la generación de valor.

Resultado 10: El 87\% de los encuestados acepta que la distribución física efectiva, es el proceso logístico que se desarrolla en torno a situar un producto en el cliente o usuario, procurando cumplir las metas, objetivos y misión de las empresas del estado.

Resultado 11: El 83\% de los encuestados acepta que la distribución física efectiva en el proceso logístico está orientado a generar valor agregado.

Análisis e interpretación de los resultados de la variable dependiente: 
Resultado 12: El 94\% de los encuestados acepta que el objetivo de la creación de valor es beneficiar al cliente o usuario y la empresa por los mayores beneficios que otorga.

Resultado 13: El 85\% de los encuestados acepta que la visión de las empresas del estado es una declaración o manifestación que indica hacia dónde se dirige o qué es aquello en lo que pretende convertirse en el largo plazo.

Resultado 14: E1 88\% de los encuestados acepta que la visión incluye el análisis de muchas de las aspiraciones de los agentes que componen las empresas del estado, tanto internos como externos

Resultado 15: El 78\% de los encuestados acepta que la misión empresarial es una declaración o manifestación duradera del objeto, propósito o razón de ser de las empresas del estado.

Resultado 16: El 94\% de los encuestados acepta que establecer la misión permite orientar las decisiones y acciones de todos los miembros de las empresas del estado en función de ésta; es decir, nos permite lograr que se establezcan objetivos, formulen estrategias y ejecuten tareas.

Resultado 17: El 90\% de los encuestados acepta que los valores empresariales son el conjunto de elementos propios que definen la estructura, línea de actuación, principios éticos y cultura organizacional de las empresas del estado.

Resultado 18: El 97\% de los encuestados acepta que los valores empresariales definen en muchos sentidos la naturaleza de las empresas, sus actuaciones y prioridades y la manera en que entienden la actividad que desarrollan para los clientes o usuarios.

Resultado 19: El 85\% de los encuestados acepta que la mejora continua empresarial se relaciona con la creatividad de recursos, actividades y procesos en las empresas del estado para generar valor.

Resultado 20: El $87 \%$ de los encuestados acepta que la mejora continua empresarial se relaciona con la innovación de los recursos, actividad y procesos empresariales. 
Resultado 21: E1 95\% de los encuestados acepta que la competitividad empresarial se relaciona con la calidad de los productos o servicios que prestan las empresas del estado.

Resultado 22: El 98\% de los encuestados acepta que la competitividad empresarial se refiere a la imposición de las empresas del estado frente a otras del propio estado como a las del sector privado.

Contrastación de la hipótesis general: La correlación $(\mathrm{R})$ Rho de Spearman es igual a $94.13 \%$, es alta, lo que significa una excelente interrelación de las variables analizadas; todo lo cual favorece en gran medida el trabajo realizado. El valor de significancia, igual a $3.22 \%$, valor obtenido que ha resultado menor al margen de error propuesto del $5.00 \%$, lo que permite aceptar la hipótesis alternativa y rechazar la hipótesis nula. La Regresión proporciona el Coeficiente de Determinación Lineal (R cuadrado) igual a $88.58 \%$; lo que estadísticamente indica el peso que tiene la variable independiente: sobre la dependiente. Se concluye que el proceso logístico efectivo influye en grado significativo en la generación de valor de las empresas del estado.

Contrastación de la hipótesis específica a) : La correlación (R) Rho de Spearman es igual a $94.23 \%$, es alta, lo que significa una excelente interrelación de las variables analizadas. El valor de significancia, igual a $2.99 \%$, valor obtenido que ha resultado menor al margen de error propuesto del $5.00 \%$, lo que permite aceptar la hipótesis alternativa. La Regresión proporciona el Coeficiente de Determinación Lineal ( $\mathrm{R}$ cuadrado) igual a $88.79 \%$; lo que estadísticamente indica el peso que tiene la variable independiente: sobre la dependiente. Se concluye que los sistemas modernos de gestión logística como la planificación de los requerimientos de materiales y la planificación de recursos de manufactura o servicios inciden en grado significativo en la generación de valor de las empresas del estado.

Contrastación de la hipótesis específica b): La correlación (R) Rho de Spearman es igual a $94.89 \%$, es alta, lo que significa una excelente interrelación de las variables analizadas. El valor de significancia, igual a 2.32\%, lo que permite rechazar la hipótesis nula. La Regresión proporciona el Coeficiente de Determinación Lineal (R cuadrado) igual a 90.06\%; lo que estadísticamente indica el peso que tiene la variable independiente: sobre la dependiente. Se concluye que las nuevas herramientas de gestión logística como 
el benchmarking y el outsourcing influyen en grado significativo en la generación de valor de las empresas del estado.

Contrastación de la hipótesis específica c): La correlación (R) Rho de Spearman es igual a 94.62\%, es alta, lo que significa una excelente interrelación de las variables analizadas. El valor de significancia, igual a 2.66\%. La Regresión proporciona el Coeficiente de Determinación Lineal ( $\mathrm{R}$ cuadrado) igual a 89.53\%; lo que estadísticamente indica el peso que tiene la variable independiente sobre la dependiente. Se concluye que los flujos productivos de la cadena logística como el método: justo a tiempo y la tecnología de producción optimizada inciden en grado significativo en la generación de valor de las empresas del estado.

Contrastación de la hipótesis específica d):

La correlación $(\mathrm{R})$ Rho de Spearman es igual a 94.07\%, es alta, lo que significa una excelente interrelación de las variables analizadas; todo lo cual favorece en gran medida el trabajo realizado. El valor de significancia, igual a $2.38 \%$, valor obtenido que ha resultado menor al margen de error propuesto del 5.00\%, lo que, de acuerdo con la teoría estadística, permite aceptar la hipótesis alternativa y desde luego rechazar la hipótesis nula; todo en el marco de la objetividad del trabajo realizado. La Regresión proporciona el Coeficiente de Determinación Lineal ( $\mathrm{R}$ cuadrado) igual a $88.45 \%$; lo que estadísticamente indica el peso que tiene la variable independiente sobre la dependiente. Se concluye que la respuesta eficiente al consumidor, la respuesta rápida y la planeación de los recursos de distribución del proceso logístico efectivo influyen en grado significativo en la generación de valor de las empresas del estado.

Contrastación de la hipótesis específica e): La correlación (R) Rho de Spearman es igual a $94.33 \%$, es alta, lo que significa una excelente interrelación. El valor de significancia igual a $2.44 \%$, lo que permite rechazar la hipótesis nula; todo en el marco de la objetividad del trabajo realizado. La Regresión proporciona el Coeficiente de Determinación Lineal ( $\mathrm{R}$ cuadrado) igual a $88.98 \%$; lo que estadísticamente indica el peso que tiene la variable independiente sobre la dependiente. Se concluye que la distribución física efectiva del proceso logístico efectivo incide en grado significativo en la generación de valor de las empresas del estado. 


\section{Discusión}

Discusión del resultado de la variable independiente: El 94\% de los encuestados acepta que el proceso logístico efectivo es el conjunto de fases que facilitan las relaciones del movimiento de los productos, tanto externa como internamente, con el objeto de cumplir la misión de las empresas del estado. Este resultado es similar al 92\% presentado, aunque en otra dimensión espacial y temporal, por Alcocer, P. y Knudsen, J. (2018). Desempeño integral de los procesos logísticos en una cadena de suministro. (Tesis de maestría). Universidad Técnica Estatal de Quevedo, Quevedo, Ecuador. Ambos resultados son altos y favorecen el modelo de investigación desarrollado.

Discusión del resultado de la variable dependiente: El 94\% de los encuestados acepta que el objetivo de la creación de valor es beneficiar al cliente o usuario y la empresa por los mayores beneficios que otorga. Este resultado es similar al $95 \%$ presentado, aunque en otra dimensión espacial y temporal, por Uruburu, A. (2013). Identificación y evaluación de la creación de valor mediante la implantación y desarrollo de políticas de responsabilidad social corporativa estratégica. (Tesis doctoral). Universidad Politécnica de Madrid. Madrid. Ambos resultados son altos y favorecen el modelo de investigación desarrollado.

\section{Conclusiones}

Conclusión general: Se ha determinado la influencia del proceso logístico efectivo en la generación de valor de las empresas del estado.

Conclusiones específicas: a) Se ha establecido la incidencia de los sistemas modernos de gestión logística como la planificación de los requerimientos de materiales y la planificación de recursos de manufactura o servicios en la generación de valor de las empresas del estado; b) Se ha determinado la influencia de las nuevas herramientas de gestión logística como el benchmarking y el outsourcing en la generación de valor de las empresas del estado; c) Se ha establecido la incidencia de los flujos productivos de la cadena logística como el método: justo a tiempo y la tecnología de producción optimizada en la generación de valor de las empresas del estado; d) Se ha determinado la influencia de la respuesta eficiente al consumidor, la respuesta rápida y la planeación de los recursos de distribución del proceso logístico efectivo en la generación de valor de las empresas 
del estado; y, e) Se ha establecido la incidencia de la distribución física efectiva del proceso logístico efectivo en la generación de valor de las empresas del estado.

\section{Recomendaciones:}

Recomendación general: Se remienda a los directivos y funcionarios tener en cuenta que el proceso logístico efectivo influye en grado significativo en la generación de valor de las empresas del estado.

Recomendaciones específicas: a) Se remienda a los directivos y funcionarios tener en cuenta que los sistemas modernos de gestión logística como la planificación de los requerimientos de materiales y la planificación de recursos de manufactura o servicios inciden en grado significativo en la generación de valor de las empresas del estado; b) Se remienda a los directivos y funcionarios tener en cuenta que las nuevas herramientas de gestión logística como el benchmarking y el outsourcing influyen en grado significativo en la generación de valor de las empresas del estado; c) Se remienda a los directivos y funcionarios tener en cuenta que los flujos productivos de la cadena logística como el método: justo a tiempo y la tecnología de producción optimizada inciden en grado significativo en la generación de valor de las empresas del estado; d) Se remienda a los directivos y funcionarios tener en cuenta que la respuesta eficiente al consumidor, la respuesta rápida y la planeación de los recursos de distribución del proceso logístico efectivo influyen en grado significativo en la generación de valor de las empresas del estado; y, e) Se remienda a los directivos y funcionarios tener en cuenta que la distribución física efectiva del proceso logístico efectivo incide en grado significativo en la generación de valor de las empresas del estado.

\section{Referencias}

Alcalde, A., García, G., Ponce, N. y Valdivieso, S. (2014). Impacto en la generación de valor en la Empresa Exalmar SAA por la falta de regulación del cese individual por causas objetivas por necesidades empresariales. Alternativa de solución. (Tesis de maestría). Universidad ESAN. Lima. Recuperada de: http://repositorio.esan.edu.pe/handle/esan/1657

Alcocer, P. y Knudsen, J. (2018). Desempeño integral de los procesos logísticos en una cadena de suministro. (Tesis de maestría). Universidad Técnica Estatal de 
Quevedo, Quevedo, Ecuador. Recuperada de: http://scielo.sld.cu/pdf/rii/v40n1/1815-5936-rii-40-01-78.pdf

Álvarez, I. (2018). Finanzas estratégicas y creación de valor. Bogotá. ECOE. Recuperado de: https://www.ecoeediciones.com/wpcontent/uploads/2016/09/finanzas-estrate\%cc\%81 gicas-y-creacio\%cc\%81n-devalor-5ta-edicio $\%$ cc\% $\% 1$ n.pdf

Bohórquez, E. y Puello, R. (2013). Diseño de un modelo de gestión logística para mejorar la eficiencia organizacional de la Empresa Coralinas \& Pisos S.A. Corpisos S.A. en el Municipio de Turbaco, Bolívar. (Tesis de maestría). Universidad de Cartagena. Cartagena, Venezuela. Recuperada de: http://190.242.62.234:8080/jspui/bitstream/11227/468/1/tesis.pdf

Bonmatí, J. (2018). El valor de una empresa y la creación de valor en esa empresa. Madrid. AECE Madrid. Recuperada de: https://dialnet.unirioja.es/descarga/articulo/3816159.pdf

Chang, J., Gamarra, G. y López, P. (2019). Valorización de la empresa unión de Cervecerías Peruanas Backus y Johnston SAA. (Tesis de maestría). Universidad del Pacífico. Lima. Recuperado de: http://repositorio.up.edu.pe/bitstream/handle/11354/2356/jes\%c3\%bas_tesis_maes tria 2019.pdf?sequence $=1$ \&isallowed $=y$

Corahua, A. y Mendoza, P. (2018). Propuesta del diseño del proceso logístico para mejorar la productividad del sector agrícola, palta Hass, en la irrigación cabeza de toro del Departamento de Ica, Perú. (Tesis de título profesional). Universidad Peruana de Ciencias Aplicadas. Lima. Recuperada de: https://repositorioacademico.upc.edu.pe/bitstream/handle/10757/625125/corahua ba.pdf? sequence $=5 \&$ isallowed $=\mathrm{y}$

Huamán, O. y Rodríguez, A. (2018). Desarrollo de una propuesta de mejora de los procesos logísticos de almacén e inventarios de insumos del Hospital Nacional Hipólito Unanue Lima, Perú. (Tesis de grado). Universidad Privada del Norte. Lima. Recuperada de: http://repositorio.upn.edu.pe/bitstream/handle/11537/14440/oscar\%20alex\%20hua 
man $\% 20$ rivera $\% 20$ -

$\% 20$ arturo $\% 20$ manuel $\% 20$ rodriguez $\% 20$ gutierrez.pdf? sequence $=1 \&$ isallowed $=y$

Marqués, M. (2017). Sostenibilidad, comunicación y valor compartido: el discurso actual del desarrollo sostenible en la empresa española. (Tesis doctoral). Universidad Complutense de Madrid. Madrid. Recuperada de: https://eprints.ucm.es/42084/1/t38628.pdf

Mora, L. (2017). Gestión Logística Integral. Bogotá. ECOE.

Ojeda, J., Quintero, J. y Machado, I (2017). La ética en la investigación. (Artículo científico). Universidad Rafael Belloso Chacín. Telos. Revista de Estudios Interdisciplinarios en Ciencias Sociales. Recuperada de: https://www.redalyc.org/pdf/993/99318750010.pdf

Orna, J. (2015). Creación de valor en la empresa: La estrategia continua. Lima. Quipukamayoc Revista de la Facultad de Ciencias Contables. Vol. 23 N. ${ }^{\circ} 43$ pp. 103-109 (2015) UNMSM, ISSN: 1560-9103 (versión impresa) / ISSN: 1609-8196 (versión electrónica).

Recuperada

de: revistasinvestigacion.unmsm.edu.pe/index.php/quipu/article/.../10398

Parodi, L. (2017). Gestión administrativa y la gestión logística del Hospital de Chancay, año 2016. (Tesis de maestría). Universidad Cesar Vallejo. Lima. Recuperada de: http://repositorio.ucv.edu.pe/bitstream/handle/ucv/8874/parodi_zlf.pdf?sequence= $\underline{1 \& \text { isallowed }=\mathrm{y}}$

Quijada, V. (2018). La problemática real de las compras públicas. Lima. Recuperada de: http://www.acpuju.com/2014/06/articulo-la-problematica-real-de-las-compraspublicas/

Ramírez, J. (2018). Propuesta de mejora en la productividad de los procesos logísticos de una empresa distribuidora de libros. (Tesis de maestría). Universidad Peruana de Ciencias Aplicadas. Lima.

Rubio, S. (2018). El sistema de logística inversa en la empresa: análisis y aplicaciones. (Tesis doctoral). Universidad de Extremadura. Madrid. Recuperada de: http://biblioteca.unex.es/tesis/8477236135.pdf 
Silva, G. (2017). Control interno y procesos logísticos en el personal administrativo de la Oficina de Abastecimiento y Patrimonio del Ministerio de Agricultura y Riego, 2015. (Tesis de maestría). Universidad Cesar Vallejo. Lima. repositorio.ucv.edu.pe/bitstream/handle/ucv/9099/silva_ago.pdf?sequence=1

Small, J. (2018). Análisis de la generación de valor de las principales inmobiliarias del Perú. (Tesis doctoral). Universidad de Piura. Recuperada de: https://pirhua.udep.edu.pe/bitstream/handle/11042/3626/tsp_ae-

\section{004.pdf? sequence $=1 \&$ isallowed $=y$}

Tellez, J. (2015). Un análisis empírico de la creación de valor de las empresas que cotizan en la Bolsa Mexicana de Valores. (Tesis doctoral). Universidad de Cantabria. Santander, España. Recuperada de: https://repositorio.unican.es/xmlui/bitstream/handle/10902/6391/tesis\%20jtp.pdf

Uruburu, A. (2013). Identificación y evaluación de la creación de valor mediante la implantación y desarrollo de políticas de responsabilidad social corporativa estratégica. (Tesis doctoral). Universidad Politécnica de Madrid. Madrid. Recuperada de: http://oa.upm.es/23182/1/angel_uruburu_colsa.pdf

Valderrama, H. y Salaverry, C. (2014). Planeamiento estratégico para el desarrollo logístico del Banco de la Nación. (Tesis de maestría). Universidad del Pacífico. Lima. Recuperada de: http://repositorio.up.edu.pe/bitstream/handle/11354/2286/hortencia_tesis_maestria 2014.pdf? sequence $=1 \&$ isallowed $=y$

Velásquez, G. (2016). Gestión logística en el sector público: ¿un tema de eficiencia o de confianza? Lima. ESAN. Recuperada de: https://www.esan.edu.pe/conexion/actualidad/2016/11/21/gestion-logistica-sectorpublico-tema-eficiencia-o-confianza/

Viscarri, J. (2019). Modelo de creación de valor para el cliente. Bogotá. Universidad Politécnica de Catalunya. Barcelona, España. Recuperada de: https://upcommons.upc.edu/bitstream/handle/2117/16640/viscarri_modelo_creaci on valor cliente.pdf 